HydroTherapy in der Wundversorgung

\section{Effektives Débridement ohne Skalpell}

— Das autolytische Débridement - im Rahmen der HydroTherapy mit dem Wundkissen HydroClean ${ }^{\circledast}$ vorgenommen - ist eine sanfte und schonende Alternative zum chirurgischen Débridement. Das supraabsorbierende Wundkissen verfügt als einzige Wundauflage über einen einzigartigen Saug-Spül-Mechanismus. HydroClean ${ }^{\oplus}$ führt der Wunde durch kontinuierliches Spülen mit Ringerlösung Feuchtigkeit zu, löst selektiv Nekrosen, Exsudat, Fibrin und Bakterien, nimmt diese auf und bindet sie sicher im Kern. Proteolytische Enzyme und Phagozyten werden aktiviert. Durch die Beseitigung von Keimreservoiren und die Verringerung der Keimkonzentration wird das Infektionsrisiko gesenkt, die Beseitigung von Gewebebarrieren und die Reduzierung von Matrixmetalloproteasen (MMP) fördert die Granulation und Epithelisierung der Wunde. Auf diese Weise schafft das Wundkissen optimale Wundverhältnisse für eine effiziente Wundheilung, die in der Granulations- und Epithelisierungsphase mit dem mit Hydrogel beschichteten Schaumverband HydroTac ${ }^{\circledast}$ weiter unterstützt werden kann.

Das autolytische Débridement mit dem Wundkissen verfüge in der praktischen Anwendung über eine Reihe weiterer Vorteile, berichtete Burkhard Jendrusch, Krankenpfleger und geprüfter Wundberater aus Westerstede: „HydroClean ${ }^{\circledast}$ kann bis zu drei Tage getragen werden; Silikonstreifen verhindern ein Verkleben mit der Wunde und sorgen für einen schmerzarmen und gewebeschonenden Verbandswechsel; dank einer visuellen Applikationshilfe ist das Kissen, das auch unter Kompression angewendet werden kann, einfach anzulegen."

(koc)

Vortrag „Was wäre, wenn Sie auch ohne Skalpell wirksam debridieren könnten?"; Deutscher Wundkongress Bremen, 11. Mai 2017 (Veranstalter: Paul Hartmann AG)

\title{
Sauberkeit und Hygiene
}

\section{Mit Dampfreiniger im Pflegeheim}

— Dass Sauberkeit und Top-Hygiene im Seniorenheim nicht auf Kosten der Betreuung der Bewohner gehen müssen, beweist das Ludwig-Steil-Haus des Evangelischen Johanneswerks in Herne: Seit die Mitarbeiter bei der Reinigung von Zimmern, Gemeinschaftsräumen, Sanitäranlagen und in der Küche die natürliche Reinigungskraft des innovativen Dampfsaugsystems Blue

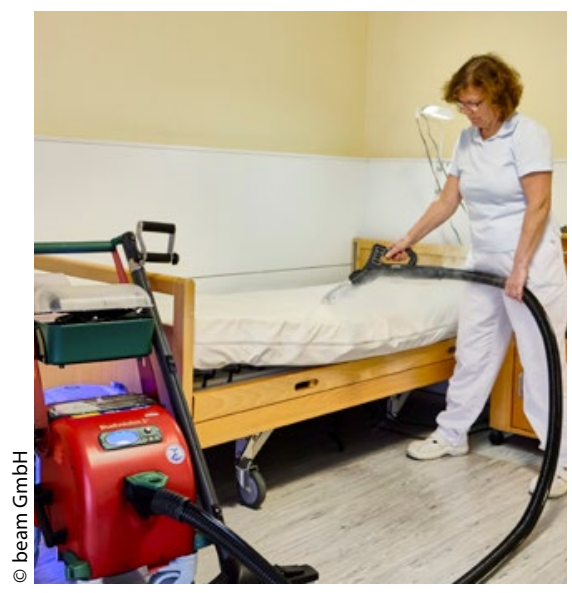

Evolution S+ der beam GmbH einsetzen, haben sie wieder mehr Zeit für ihre Patienten. Der effiziente, HACCP-zertifizierte Saubermacher dampft und saugt in einem Arbeitsgang und spart dem Reinigungsteam damit bis zu $60 \%$ der Zeit, die bislang fürs Saubermachen nötig war. So haben die Mitarbeiter wieder mehr Zeit für die Bewohner und gleichzeitig auch die Hygiene im Haus fest im Griff. Weiteres Plus: Die Geräte der Blue-Evolution-Serie arbeiten ausschließlich mit heißem Wasserdampf und verzichten komplett auf chemische Reinigungsmittel.

Als Besonderheit wartete der Blue Evolution zudem mit einem Blaulicht-Effekt auf, bei dem Keime keine Chance haben. Denn bei jedem Saugvorgang landen die gelösten Schmutzpartikel im Wasserfilter und werden dort im Wasser gebunden. Die im Schmutzwasser enthaltenen Keime werden dann über das Blaulicht abgetötet und die Luft so effizient mitgereinigt.

www.beam.de
Hautpflege

\section{Handekzem und} Neurodermitis

— Der häufige Kontakt der Hände mit Wasser, Desinfektionsmittel und Reinigungsmitteln oder anderen Irritantien kann die Hautbarriere schädigen. Als Folge neigen die Hände verstärkt zu Trockenheit, Rötungen, kleineren Einrissen, Juckreiz und Irritationen. Die AtopiControl Hand Intensiv-Creme glättet, regeneriert und beruhigt spürbar die Haut - sie wird widerstandsfähiger und die Belastbarkeit auch bei häufigem Händewaschen wird erhöht. Bei regelmäßiger Anwendung verbessert sich der Hautzustand nachhaltig. Die schnell einziehende, nicht fettende Öl-in-Wasser-Creme enthält verschiedene Pflegewirkstoffe auf pflanzlicher Basis, darunter: Licochalcone A, ein Extrakt aus der Süßholzwurzel, der die Haut beruhigt. Ceramide regenerieren die Haut und helfen ihr, die natürliche Hautbarriere wiederherzustellen. Menthoxypropanediol, ein Abkömmling von Menthol, hat kühlende und Juckreiz lindernde Eigenschaften. Die Hand Intensiv-Creme kann auch als Basis-Pflege bei Neurodermitis in der schubfreien Zeit sowie als Intensivpflege in der akuten Phase angewendet werden.

Hinweis für Allergiker: Die Handcreme enthält Hafermehl. Es stärkt die natürliche Hautbarriere, fördert die Heilung von Verletzungen und lindert Hautreizungen.

Die Produktserie AtopiControl besteht aus einer Basispflege für die schubfreien Phasen und einer Intensivpflege für die akuten Phasen. Hier beruhigt und lindert sie Juckreiz und verbessert den Hautzustand. Die Systempflege enthält keine Duft- und Farbstoffe oder Parabene. Die Produkte sind auch für Kinder und Babys mit Neurodermitis ab drei Monaten gut verträglich. Nur das Eucerin AtopiControl Anti-Juckreiz Spray wird erst für Kinder ab drei Jahren empfohlen.

www.eucerin.de 